\title{
Dualisation of the symmetric space sigma model with couplings
}

\author{
Tekin Dereli ${ }^{a}$, Nejat T. Y1lmaz ${ }^{\mathrm{b}}$ \\ ${ }^{a}$ Department of Physics, Koç University, Rumelifeneri Yolu 34450, Sartyer, Istanbul, Turkey \\ ${ }^{\mathrm{b}}$ Department of Mathematics and Computer Sciences, Çankaya University, \\ Ö $\breve{g r e t m e n l e r ~ C a d . ~ N o: ~} 14$ 06530, Balgat, Ankara, Turkey \\ Received 5 May 2004; accepted 9 November 2004 \\ Available online 25 November 2004
}

\begin{abstract}
The first-order formulation of the $G / K$ symmetric space sigma model of the scalar cosets of the supergravity theories is discussed when there is coupling of $(m-1)$-form matter fields. The Lie superalgebra which enables the dualized coset formulation is constructed for a general scalar coset $G / K$ with matter coupling where $G$ is a non-compact real form of a semi-simple Lie group and $K$ is its maximal compact subgroup.

(C) 2004 Elsevier B.V. All rights reserved.
\end{abstract}

\section{Introduction}

The non-linear nature of the scalar sectors of the maximal supergravities has been enlarged to formulate the non-gravitational bosonic field equations as non-linear realizations in $[1,2]$. The coset formulation of the scalars is improved to cover the other bosonic fields as well. The method of [1,2] includes the dualisation of the field content and the construction of a Lie superalgebra which generates the doubled coset element whose Cartan form would lead to the original field equations by satisfying the Cartan-Maurer equation. After

E-mail addresses: tdereli@ku.edu.tr (T. Dereli), ntyilmaz@cankaya.edu.tr (N.T. Y1lmaz). 
the determination of the algebra structure it is possible to express the first-order field equations as a twisted self-duality condition which the dualized Cartan form satisfies. In [3-5] a more general coset formulation of the IIA [6-8], the IIB [9-11] and the $D=11$ [12] supergravity theories is introduced to include the gravity as well.

The scalar sectors of a wide class of supergravities, in particular the scalar sectors of all the pure and the matter coupled $N>2$ extended supergravities in $D=4,5,6,7,8,9$ dimensions as well as the maximally extended supergravities in $D \leqslant 11$ can be formulated as symmetric space sigma models. The global symmetry groups $G$ of the scalar also the bosonic sectors of the lower-dimensional Kaluza-Klein descendant supergravities of the $D=11$ supergravity (the maximal supergravities) are semi-simple split real forms (maximally non-compact). For this reason the scalar coset manifolds $G / K$ where $K$ is the maximal compact subgroup of $G$ are Riemannian globally symmetric spaces [13] and they can be parameterized by the Borel subalgebra of $G$. In general, especially for the matter coupled supergravities, the scalar coset manifolds $G / K$ are based on non-split ${ }^{1}$ global symmetry groups $G$. In this case one has to use the solvable Lie algebra gauge [14] to parameterize the Riemannian globally symmetric space scalar coset manifold $G / K$.

In [15] the $G / K$ symmetric space sigma model is discussed in detail when the global symmetry group $G$ is in general, a non-compact semi-simple real form. The dualisation and the first-order formulation of the general non-split symmetric space sigma model is also performed in [15]. In this work we consider the coupling of other fields to the scalar coset Lagrangian of the general non-split $G / K$ symmetric space sigma model. We will perform the complete dualisation of the fields and the first-order formulation when there is coupling of other $(m-1)$-form matter fields to the scalar coset $G / K$. We will construct the dualized coset element which will realize the field equations of the scalar coset which is coupled to the $(m-1)$-form fields. We will assume the most general non-split scalar coset case which is discussed in [15-17]. Beside the scalar fields there will be a number of $m$-form field strengths whose number is fixed by the dimension of the fundamental representation of the Lie algebra $\mathbf{g}_{0}$ of $G$. As it will be clear in the next section the dimension of the representation and the number of the coupling fields must be the same so that the coupling kinetic term between the scalar coset and the matter fields in the Lagrangian can be constructed within an appropriate representation of the global symmetry group $G$ $[16,17]$. We will follow the standard dualisation method of $[1,2]$ by introducing auxiliary dual fields and by assigning generators to the original and the dual fields. The first objective of this work will be to derive the Lie superalgebra structure which generates the doubled coset element. The first-order formulation will then be presented as a twisted self-duality equation $[1,2]$ by using the derived algebra structure and by calculating explicitly the doubled field strength. The dualisation method presented in $[1,2]$ is the non-linear realization of the relative supergravity theory, it is also another manifestation of the Lagrange multiplier methods in which the dual fields correspond to the Lagrange multipliers which are introduced to construct the Bianchi Lagrangians. For this reason the Cartan form which is generated by the dualized coset element, not only realizes the original second-order field

\footnotetext{
${ }^{1}$ By non-split we mean that $G$ is a non-compact real form of a semi-simple Lie group but it is not necessarily maximally non-compact (split).
} 
equations of the matter coupled scalar coset by satisfying the Cartan-Maurer equation but also yields the first-order field equations via a twisted self-duality equation $[1,2,15]$. This first-order formulation corresponds to the construction of the dualized Lagrangian by adding the Bianchi terms to the Lagrangian of the original fields and consequently to the derivation of the first-order algebraic field equations of the original fields in terms of the Lagrange multiplier (dual) fields [18].

We start by discussing the Lagrangian and deriving the field equations in Section 2. In Section 3 we work out the dualisation and we construct the algebraic structure which realizes the field equations and finally we obtain the first-order field equations.

\section{The symmetric space sigma model and the couplings}

The scalar sectors of a wide class of supergravity theories are formulated as $G / K$ symmetric space sigma models $[1,2,16,17]$. The group $G$ is the global symmetry group of the corresponding scalar Lagrangian and it is a non-compact real form of a semi-simple Lie group. The local symmetry group $K$ is the maximal compact subgroup of $G$. The coset space $G / K$ is a Riemannian globally symmetric space for all the possible $G$-invariant Riemannian structures on $G / K$ [13]. There is a legitimate parametrization of the coset representatives by using the solvable Lie algebra of $G[13,14]$. If $\mathbf{h}_{\mathbf{k}}$ is the subalgebra of the Cartan subalgebra $\mathbf{h}_{0}$ of $\mathbf{g}_{0}$ (the Lie algebra of $G$ ) which generates the maximal R-split torus in $G$ [13-16] let for $i=1, \ldots, r\left\{H_{i}\right\}$ be the generators of $\mathbf{h}_{\mathbf{k}}$ and also let $\left\{E_{m}\right\}$ be the subset of the positive root generators of $\mathbf{g}_{0}$ such that $m \in \Delta_{\mathrm{nc}}^{+}$. The roots in $\Delta_{\mathrm{nc}}^{+}$are the non-compact roots with respect to the Cartan involution $\theta$ which is induced by the Cartan decomposition

$$
\mathbf{g}_{0}=\mathbf{k}_{0} \oplus \mathbf{u}_{0},
$$

where $\mathbf{k}_{0}$ is the Lie algebra of $K$ and $\mathbf{u}_{0}$ is a vector subspace of $\mathbf{g}_{0}[13,15]$. The positive root generators $\left\{E_{m}\right\}$ generate a nilpotent Lie subalgebra $\mathbf{n}_{\mathbf{k}}$ of $\mathbf{g}_{0}$ [16]. The coset representatives of $G / K$ which are the image points of the map from the $D$-dimensional spacetime (we assume $D>2$ in order that the dualisation analysis of the next section would be meaningful and we will take the signature of the spacetime as $(-,+,+, \ldots))$ into the group $G$ can be expressed as

$$
v(x)=e^{\frac{1}{2} \phi^{i}(x) H_{i}} e^{\chi^{m}(x) E_{m}} .
$$

This is called the solvable Lie algebra parametrization [14]. We should state that we make use of the Iwasawa decomposition

$$
\mathbf{g}_{0}=\mathbf{k}_{0} \oplus \mathbf{s}_{0}=\mathbf{k}_{0} \oplus \mathbf{h}_{\mathbf{k}} \oplus \mathbf{n}_{\mathbf{k}},
$$

where $\mathbf{s}_{0}$ is the solvable Lie subalgebra of $\mathbf{g}_{0}$ which is isomorphic to $\mathbf{u}_{0}$ as a vector space $[13,15]$. The diffeomorphism from $\mathbf{u}_{0}$ onto the Riemannian globally symmetric space $G / K$ [13] enables the construction of the parametrization in (2.2).

An involutive automorphism $\theta \in \operatorname{Aut}\left(\mathbf{g}_{0}\right)$ of a semi-simple real Lie algebra $\mathbf{g}_{0}$ is called a Cartan involution if the induced bilinear form $B_{\theta}(X, Y)=-B(X, \theta(Y))$ where $B$ is the 
Killing form on $\mathbf{g}_{0}$ is strictly positive definite $\forall X, Y \in \mathbf{g}_{0}$. If the semi-simple complex Lie algebra $\mathbf{g}=\mathbf{g}_{0}^{C}$ is the complexification of $\mathbf{g}_{0}$ then the set of elements $\mathbf{t}$ of $\mathbf{g}$ which is generated as

$$
\mathbf{t}=\mathbf{k}_{0}+i \mathbf{u}_{0}
$$

through the complexification of $\mathbf{g}_{0}$, is a compact real form of $\mathbf{g}$ whose conjugation will be denoted by $\tau$. We should bear in mind that $\mathbf{g}_{0}$ has the set equivalent images in $\mathbf{g}=\mathbf{g}_{0}^{C}$ whose realizations in $\mathbf{g}_{0} \times \mathbf{g}_{0}$ are isomorphic to $\mathbf{g}_{0}$. In this way $\mathbf{k}_{0}$ and $\mathbf{u}_{0}$ can be considered as subsets of $\mathbf{g}$ and then $\mathbf{t}$ which is a subset of $\mathbf{g}$ is also a subset of one of the images of $\mathbf{g}_{0}$ in $\mathbf{g}$. Thus under the realization of $\mathbf{g}, \mathbf{t}^{R}$ corresponds to a subalgebra of $\mathbf{g}_{0}$. The real semisimple Lie algebra $\mathbf{g}_{0}$ is also a real form of its complexification $\mathbf{g}$ so that we may define $\sigma$ as the conjugation of $\mathbf{g}$ with respect to $\mathbf{g}_{0}$. The map $\theta=\sigma \cdot \tau=\tau \cdot \sigma$ is an involutive automorphism of $\mathbf{g}$. In fact $\theta$ is a Cartan involution of $\mathbf{g}$. The $\mathbb{R}$-linear restriction of $\theta$ on the image of $\mathbf{g}_{0}$ in $\mathbf{g}$ induces a Cartan involution on $\mathbf{g}_{0}$ which we will again denote by $\theta$. After the introduction of the Cartan involution $\theta$ we can easily define the roots in $\Delta_{\mathrm{nc}}^{+}$. For each element $\alpha \in \mathbf{h}_{0}^{*}$ the dual space of the Cartan subalgebra $\mathbf{h}_{0}$ of $\mathbf{g}_{0}$ we can define the element $\alpha^{\theta} \in \mathbf{h}_{0}^{*}$ such that $\alpha^{\theta}(H)=\alpha(\theta(H)), \forall H \in \mathbf{h}_{0}$. If $\alpha \in \Delta$ then $\alpha^{\theta} \in \Delta$ as well. Thus we have defined

$$
\Delta_{\mathrm{nc}}^{+}=\left\{\alpha \mid \alpha \in \Delta^{+}, \alpha \neq \alpha^{\theta}\right\} .
$$

The scalar Lagrangian is defined in terms of the internal metric $\mathcal{M}=v^{\#} v$ where we have introduced the generalized transpose \# which is over the Lie group $G$ such that $(\exp (g))^{\#}=$ $\exp \left(g^{\#}\right) \forall g \in \mathbf{g}_{0}$. It is induced by the Cartan involution $\theta$ over the Lie algebra $\mathbf{g}_{0}$ of $G$ $\left(g^{\#}=-\theta(g)\right)[1,15,17]$. Thus in terms of the internal metric $\mathcal{M}$ the globally $G$-invariant and the locally $K$-invariant scalar Lagrangian $[1,2]$ is

$$
\mathcal{L}_{\text {scalar }}=\frac{1}{4} \operatorname{tr}\left(d \mathcal{M}^{-1} \wedge * d \mathcal{M}\right) .
$$

The $G / K$ symmetric space sigma model is studied in detail in [15]. Thus referring to [15] we can calculate the Cartan form $\mathcal{G}_{0}=d v v^{-1}$ generated by the map (2.2) as

$$
\mathcal{G}_{0}=\frac{1}{2} d \phi^{i} H_{i}+\overrightarrow{\mathbf{E}^{\prime}} \boldsymbol{\Omega} \overrightarrow{d \chi}
$$

We have used that $\left[H_{i}, E_{\alpha}\right]=\alpha_{i} E_{\alpha}$. The row vector $\overrightarrow{\mathbf{E}}^{\prime}$ has the components $\left(\overrightarrow{\mathbf{E}}^{\prime}\right)_{\alpha}=$ $e^{\frac{1}{2} \alpha_{i} \phi^{i}} E_{\alpha}$. The column vector $\overrightarrow{d \chi}$ is $\left(d \chi^{\alpha}\right)$. We have also defined the matrix $\boldsymbol{\Omega}$ as

$$
\boldsymbol{\Omega}=\sum_{n=0}^{\infty} \frac{\omega^{n}}{(n+1) !}=\left(e^{\omega}-I\right) \omega^{-1},
$$

where $\omega_{\beta}^{\gamma}=\chi^{\alpha} K_{\alpha \beta}^{\gamma}$ with the structure constants $K_{\alpha \beta}^{\gamma}$ defined as $\left[E_{\alpha}, E_{\beta}\right]=K_{\alpha \beta}^{\gamma} E_{\gamma}$. Here both $\Omega$ and $\omega$ are $n \times n$ matrices where $n$ is the number of the roots in $\Delta_{\mathrm{nc}}^{+}$[15].

We will consider the coupling of $(m-1)$-form potential fields $\left\{A^{l}\right\}$ to the $G / K$ scalar coset where the number of the coupling fields is determined such that they form a fundamental representation of $\mathbf{g}_{0}$. The quadratic terms due to this coupling which must be added 
to the scalar Lagrangian (2.6) are the combinations of the internal metric $\mathcal{M}$ and the field strengths $F^{l}=d A^{l}$

$$
\mathcal{L}_{m}=-\frac{1}{2} \mathcal{M}_{k l} F^{k} \wedge * F^{l}=-\frac{1}{2} F \wedge \mathcal{M} * F .
$$

As it is clear from above $\mathcal{M}$ and $v$ are in an appropriate representation (i.e., fundamental representation of $\mathbf{g}_{0}$ ) which is compatible with the number of the coupling fields. Thus the total Lagrangian becomes

$$
\mathcal{L}=\frac{1}{4} \operatorname{tr}\left(d \mathcal{M}^{-1} \wedge * d \mathcal{M}\right)-\frac{1}{2} F \wedge \mathcal{M} * F
$$

The Cartan involution $\theta$ induced by the Cartan decomposition (2.1) is an involutive automorphism of $\mathbf{g}_{0}$ for this reason it has two eigenspaces $\theta^{+}, \theta^{-}$with eigenvalues \pm 1 . The Cartan involution $\theta$ induces the eigenspace decomposition of the Lie algebra $\mathbf{g}_{0}$ as

$$
\mathbf{g}_{0}=\theta^{+} \oplus \theta^{-} \text {. }
$$

The elements of $\theta^{+}$are called compact while the elements of $\theta^{-}$are called non-compact. If the subgroup of $G$ generated by the compact generators is an orthogonal group then in the fundamental representation the generators can be chosen such that $\mathbf{g}^{\#}=\mathbf{g}^{T}$. Therefore \# coincides with the ordinary matrix transpose and $\mathcal{M}$ becomes a symmetric matrix in the representation we choose. We will assume this case in our further analysis bearing in mind that for the general case higher-dimensional representations are possible in which we can still take $\mathbf{g}^{\#}=\mathbf{g}^{T}[17]$.

By following the analysis of [15-17] and by using (2.7) we can derive the field equations for the coupling potentials $\left\{A^{k}\right\}$, the axions $\left\{\chi^{m}\right\}$ and the dilatons $\left\{\phi^{i}\right\}$ of the Lagrangian (2.10). Thus the corresponding field equations are

$$
\begin{aligned}
d\left(\mathcal{M}_{k l} * F^{l}\right)= & 0, \\
d\left(e^{\frac{1}{2} \gamma_{i} \phi^{i}} * U^{\gamma}\right)= & -\frac{1}{2} \gamma_{j} e^{\frac{1}{2} \gamma_{i} \phi^{i}} d \phi^{j} \wedge * U^{\gamma} \\
& +\sum_{\alpha-\beta=-\gamma} e^{\frac{1}{2} \alpha_{i} \phi^{i}} e^{\frac{1}{2} \beta_{i} \phi^{i}} N_{\alpha,-\beta} U^{\alpha} \wedge * U^{\beta}, \\
d\left(* d \phi^{i}\right)= & \frac{1}{2} \sum_{\alpha \in \Delta_{\mathrm{nc}}^{+}} \alpha_{i} e^{\frac{1}{2} \alpha_{i} \phi^{i}} U^{\alpha} \wedge e^{\frac{1}{2} \alpha_{i} \phi^{i}} * U^{\alpha} \\
& +(-1)^{D+1} \frac{1}{2}\left(\left(H_{i}\right)_{n l} \nu_{m}^{n} \nu_{j}^{l}\right) F^{j} \wedge * F^{m},
\end{aligned}
$$

where $i, j=1, \ldots, r$ and $\alpha, \beta, \gamma \in \Delta_{\mathrm{nc}}^{+}$. The roots in $\Delta_{\mathrm{nc}}^{+}$and their corresponding generators $\left\{E_{m}\right\}$ are assumed to be enumerated. We have also defined the vector $U^{\alpha}=\boldsymbol{\Omega}_{\beta}^{\alpha} d \chi^{\beta}$. Furthermore the matrices $\left\{\left(H_{i}\right)_{n l}\right\}$ are the representatives of the Cartan generators $\left\{H_{i}\right\}$ under the representation chosen. We use the notation $\left[E_{\alpha}, E_{\beta}\right]=N_{\alpha, \beta} E_{\alpha+\beta}$. We should remark that in the dilaton equation in (2.12) the contribution from the coupling fields $\left\{A^{k}\right\}$ is expressed in terms of the original fields rather than their weight expansions unlike the expressions in $[16,17]$. For notational convenience we raise or lower the indices of the matrices by using an Euclidean metric. 


\section{Dualisation and the first-order formulation}

In this section we will adopt the method of [1,2] to establish a coset formulation and to derive the first-order field equations for the Lagrangian (2.10). Basically we will improve the analysis presented for the non-split scalar coset in [15] to the case when there is matter field coupling to the non-split scalar coset. We will first define a Lie superalgebra which will realize the doubled coset element. We assign the generators $\left\{H_{i}, E_{m}, V_{j}\right\}$ to the fields $\left\{\phi^{i}, \chi^{m}, A^{j}\right\}$, respectively. We assume that $\left\{H_{i}, E_{m}\right\}$ are even generators within the superalgebra structure since the coupling fields are scalars and they have even rank. The generators $\left\{V_{j}\right\}$ are even or odd whether the rank of the coupling fields $\left\{A^{j}\right\}$ namely $(m-1)$ is even or odd. The next step is to introduce the dual fields $\left\{\tilde{\phi}^{i}, \tilde{\chi}^{m}, \tilde{A}^{j}\right\}$ which would arise as a result of the local integration of the field equations (2.12). The first two are $(D-2)$-forms and the last ones are $(D-m-1)$-forms. We also assign the dual generators $\left\{\tilde{H}_{i}, \tilde{E}_{m}, \tilde{V}_{j}\right\}$ to these dual fields, respectively. The dual generators are even or odd depending on $D$ and $m$ in other words according to the rank of the dual fields they are assigned to. We will derive the structure of the Lie superalgebra generated by the original and the dual generators we have introduced so that it will enable a coset formulation for the Lagrangian (2.10). Similar to the non-linear coset structure of the scalars presented in the last section we can define the map

$$
v^{\prime}=e^{\frac{1}{2} \phi^{i} H_{i}} e^{\chi^{m} E_{m}} e^{A^{j} V_{j}} e^{\tilde{A}^{j} \tilde{V}_{j}} e^{\tilde{\chi}^{m} \tilde{E}_{m}} e^{\frac{1}{2} \tilde{\phi}^{i} \tilde{H}_{i}},
$$

which can be considered as the parametrization of a coset via the differential graded algebra [2] generated by the differential forms on the $D$-dimensional spacetime and the Lie superalgebra of the original and the dual generators we propose. We are not intending to detect the group theoretical structure of this coset, rather we will only aim to construct the Lie superalgebra of the original and the dual generators which function in the parametrization (3.1). If one knows the structure constants of this algebra one can calculate the Cartan form $\mathcal{G}^{\prime}=d v^{\prime} v^{\prime-1}$ which is induced by the map (3.1). Due to its definition the Cartan form $\mathcal{G}^{\prime}$ obeys the Cartan-Maurer equation

$$
d \mathcal{G}^{\prime}-\mathcal{G}^{\prime} \wedge \mathcal{G}^{\prime}=0
$$

By following the outline of [1,2] the structure constants of the Lie superalgebra will be chosen so that when we calculate the Cartan form $\mathcal{G}^{\prime}$ it will lead us to the second-order field equations (2.12) via the identity (3.2) and it will satisfy the twisted self-duality equation $* \mathcal{G}^{\prime}=\mathcal{S G}^{\prime}$ where the action of the pseudo-involution $\mathcal{S}$ [2] on the generators is taken as

$$
\begin{array}{ll}
\mathcal{S} H_{i}=\tilde{H}_{i}, & \mathcal{S} E_{m}=\tilde{E}_{m}, \quad \mathcal{S} \tilde{E}_{m}=(-1)^{D} E_{m}, \quad \mathcal{S} \tilde{H}_{i}=(-1)^{D} H_{i}, \\
\mathcal{S} V_{j}=\tilde{V}_{j}, & \mathcal{S} \tilde{V}_{j}=(-1)^{m(D-m)+1} V_{j} .
\end{array}
$$

We know that the twisted self-duality equation will give us the locally integrated firstorder field equations which can be obtained from (2.12) by extracting an overall exterior derivative operator on both sides of the equations $[1,2,15,18]$. This local integration produces auxiliary fields which are the dual fields we introduce. The dualisation method is nothing but another manifestation of the Lagrange multiplier method while the dual fields correspond to the Lagrange multiplier fields which are introduced to construct the Lagrange multiplier Lagrangian terms of the Bianchi identities of the original field strengths 
[18]. We may first calculate the Cartan form $\mathcal{G}^{\prime}=d v^{\prime} v^{\prime-1}$ from the map (3.1) in terms of the unknown structure constants of the Lie superalgebra of the original and the dual generators. We intend to construct an algebraic structure so that the Cartan form satisfies the twisted self-duality equation $* \mathcal{G}^{\prime}=\mathcal{S G}^{\prime}$. In a sense the twisted self-duality equation would correspond to the equation of motion of the dualized Lagrangian [2]. At this stage we will assume that the Lie superalgebra of the original and the dual generators has a general structure in which the commutator or the anti-commutator of two original generators gives another original generator, an original and a dual generator leads to a dual generator while two dual generators vanish under the algebra product. When we calculate the structure constants of the Lie superalgebra which generates the correct Cartan form $\mathcal{G}^{\prime}$ which leads to the field equations (2.12) in (3.2) we will see that they obey such a general scheme indeed. We may use the proposed twisted self-duality property of the dualized Cartan form primarily to write it only in terms of the original fields because as it is clear from (3.3) the pseudo-involution sends the original generators to the dual ones and the dual ones to the originals with a sign factor. Thus by using the formulas

$$
\begin{aligned}
& d e^{X} e^{-X}=d X+\frac{1}{2 !}[X, d X]+\frac{1}{3 !}[X,[X, d X]]+\cdots, \\
& e^{X} Y e^{-X}=Y+[X, Y]+\frac{1}{2 !}[X,[X, Y]]+\cdots,
\end{aligned}
$$

effectively and by applying the twisted self-duality condition $* \mathcal{G}^{\prime}=\mathcal{S G}^{\prime}$, the calculation of the Cartan form $\mathcal{G}^{\prime}=d v^{\prime} v^{\prime-1}$ only in terms of the original fields yields

$$
\begin{aligned}
\mathcal{G}^{\prime}= & \frac{1}{2} d \phi^{i} H_{i}+\overrightarrow{\mathbf{E}^{\prime}} \boldsymbol{\Omega} \overrightarrow{d \chi}+\overrightarrow{\mathbf{V}} e^{\mathbf{U}} e^{\mathbf{B}} \overrightarrow{\mathbf{d A}}+\frac{1}{2}(-1)^{D} * d \phi^{i} \tilde{H}_{i} \\
& +(-1)^{D} e^{\frac{1}{2} \alpha_{i} \phi^{i}} \boldsymbol{\Omega}_{\beta}^{\alpha} * d \chi^{\beta} \tilde{E}_{\alpha}+(-1)^{(m(D-m)+1)} \overrightarrow{\tilde{\mathbf{V}}} e^{\mathbf{U}} e^{\mathbf{B}} * \overrightarrow{\mathbf{d} \mathbf{A}} .
\end{aligned}
$$

We have defined the yet unknown structure constants as

$$
\left[H_{i}, V_{n}\right]=\theta_{i n}^{t} V_{t}, \quad\left[E_{m}, V_{j}\right]=\beta_{m j}^{l} V_{l} .
$$

The matrices $\mathbf{U}$ and $\mathbf{B}$ in (3.5) are

$$
(\mathbf{U})_{v}^{n}=\frac{1}{2} \phi^{i} \theta_{i v}^{n}, \quad(\mathbf{B})_{n}^{j}=\chi^{m} \beta_{m n}^{j} .
$$

We introduce the row vectors $\overrightarrow{\mathbf{V}}$ and $\overrightarrow{\tilde{\mathbf{V}}}$ as $\left(V_{i}\right)$ and $\left(\tilde{V}_{j}\right)$, respectively, the column vector $\overrightarrow{\mathbf{d A}}$ is $\left(d A^{i}\right)$. We have also taken

$$
\left[V_{m}, V_{n}\right\}=0 .
$$

In (3.5) we have made use of the results of [15] in the calculation of the scalar sector of the Cartan form $\mathcal{G}^{\prime}=d v^{\prime} v^{\prime-1}$.

Now inserting the Cartan form (3.5) (which is written only in terms of the original fields by primarily applying the twisted self-duality condition) in the Cartan-Maurer identity (3.2) should result in the second-order field equations $(2.12)[2,15]$. This main feature of the coset formulation enables us to derive the commutation and the anti-commutation 
relations of the original generators which are already encoded in (3.5) and the commutators and the anti-commutators of the dual and the mixed (an original and a dual) generators which arise in the calculation of (3.2) within the graded differential algebra structure of the differential forms and the generators. Thus a straightforward calculation of (3.2) by inserting (3.5) and then the comparison of the result with the second-order field equations (2.12) gives us the desired structure constants of the commutators and the anti-commutators. We have

$$
\begin{aligned}
& {\left[H_{j}, E_{\alpha}\right]=\alpha_{j} E_{\alpha}, \quad\left[E_{\alpha}, E_{\beta}\right]=N_{\alpha, \beta} E_{\alpha+\beta},} \\
& {\left[H_{l}, V_{i}\right]=\left(H_{l}\right)_{i}^{k} V_{k}, \quad\left[E_{\alpha}, V_{i}\right]=\left(E_{\alpha}\right)_{i}^{j} V_{j},} \\
& {\left[H_{j}, \tilde{E}_{\alpha}\right]=-\alpha_{j} \tilde{E}_{\alpha}, \quad\left[E_{\alpha}, \tilde{E}_{\alpha}\right]=\frac{1}{4} \sum_{j=1}^{r} \alpha_{j} \tilde{H}_{j},} \\
& {\left[E_{\alpha}, \tilde{E}_{\beta}\right]=N_{\alpha,-\beta} \tilde{E}_{\gamma}, \quad \alpha-\beta=-\gamma, \alpha \neq \beta,} \\
& {\left[H_{i}, \tilde{V}_{k}\right]=-\left(H_{i}^{T}\right)_{k}^{l} \tilde{V}_{l}, \quad\left[E_{\alpha}, \tilde{V}_{k}\right]=-\left(E_{\alpha}^{T}\right)_{k}^{l} \tilde{V}_{l},} \\
& {\left[V_{l}, \tilde{V}_{k}\right]=(-1)^{D-m} \frac{1}{4} \sum_{i}\left(H_{i}\right)_{l k} \tilde{H}_{i},}
\end{aligned}
$$

where the indices of the Cartan generators and their duals are $i, j, l=1, \ldots, r$ and $\alpha, \beta, \gamma \in$ $\Delta_{\mathrm{nc}}^{+}$. The matrices $\left(\left(E_{\alpha}\right)_{i}^{j},\left(H_{l}\right)_{i}^{j}\right)$ above are the representatives of the corresponding generators $\left(\left(E_{\alpha}\right),\left(H_{l}\right)\right)$. Also $\left(\left(E_{\alpha}^{T}\right)_{i}^{j},\left(H_{l}^{T}\right)_{i}^{j}\right)$ are the matrix transpose of $\left(\left(E_{\alpha}\right)_{i}^{j},\left(H_{l}\right)_{i}^{j}\right)$. We should state once more that the dimension of the matrices above namely the dimension of the fundamental representation of $\mathbf{g}_{0}$ is equal to the number of the coupling fields and their corresponding generators since this is how we have defined and constructed the coupling of the matter fields $A^{k}$ to the scalar coset $G / K$ in the Lagrangian (2.10). The remaining commutators or the anti-commutators of the original and the dual generators which are not listed in (3.9) vanish indeed. We observe that as we have assumed before the Lie superalgebra we have constructed in (3.9) has the general form

$$
\begin{aligned}
& {[O, \tilde{D}\} \subset \tilde{D}, \quad[O, O\} \subset O,} \\
& {[\tilde{D}, \tilde{D}\}=0,}
\end{aligned}
$$

where $O$ is the set of the original and $\tilde{D}$ is the set of the dual generators.

Now that we have determined the structure constants of the algebra generated by the original and the dual generators we can explicitly calculate the Cartan form $\mathcal{G}^{\prime}=d v^{\prime} v^{\prime-1}$ in terms of both the original and the dual fields. By using the identities in (3.4) also the structure constants given in (3.9) effectively we have

$$
\begin{aligned}
\mathcal{G}^{\prime}= & \frac{1}{2} d \phi^{i} H_{i}+\overrightarrow{\mathbf{E}^{\prime}} \boldsymbol{\Omega} \overrightarrow{d \chi}+\overrightarrow{\tilde{\mathbf{T}}} e^{\boldsymbol{\Gamma}} e^{\boldsymbol{\Lambda}} \overrightarrow{\tilde{\mathbf{S}}}+\overrightarrow{\mathbf{V}} v \overrightarrow{\mathbf{d} \mathbf{A}}+\overrightarrow{\tilde{\mathbf{V}}}\left(v^{T}\right)^{-1} \overrightarrow{\mathbf{d} \tilde{\mathbf{A}}} \\
& +(-1)^{m(D-m)} \sum_{i=1}^{r} \frac{1}{4}\left(H_{i}\right)_{k l} A^{k} \wedge d \tilde{A}^{l} \tilde{H}_{i} .
\end{aligned}
$$

In addition to the definitions given in Section 2 we have introduced the row vectors $\overrightarrow{\mathbf{V}}$ and $\overrightarrow{\tilde{\mathbf{V}}}$ as $\left(V_{k}\right)$ and $\left(\tilde{V}_{l}\right)$, respectively. The column vectors $\overrightarrow{\mathbf{d A}}$ and $\overrightarrow{\mathbf{d} \tilde{\mathbf{A}}}$ are $\left(F^{k}\right)$ and $\left(d \tilde{A}^{l}\right)$. 
Besides we have the row vector of the duals of the solvable Lie algebra generators of $G$ as $\tilde{\mathbf{T}}_{i}=\tilde{H}_{i}$ for $i=1, \ldots, r$ and $\tilde{\mathbf{T}}_{r+\alpha}=\tilde{E}_{\alpha}$ for $\alpha \in \Delta_{\text {nc }}^{+}$. The column vector $\overrightarrow{\tilde{\mathbf{S}}}$ is defined as $\tilde{\mathbf{S}}^{i}=\frac{1}{2} d \tilde{\phi}^{i}$ for $i=1, \ldots, r$ and $\tilde{\mathbf{S}}^{r+\alpha}=d \tilde{\chi}^{\alpha}$ for $\alpha \in \Delta_{\mathrm{nc}}^{+}$. We have introduced the matrices $\Gamma$ and $\boldsymbol{\Lambda}$ as

$$
\boldsymbol{\Gamma}_{n}^{k}=\frac{1}{2} \phi^{i} \tilde{g}_{i n}^{k}, \quad \Lambda_{n}^{k}=\chi^{m} \tilde{f}_{m n}^{k}
$$

Here we have used the structure constants $\left\{\tilde{g}_{i n}^{k}\right\}$ and $\left\{\tilde{f}_{m n}^{k}\right\}$ from their definitions in

$$
\left[E_{\alpha}, \tilde{T}_{m}\right]=\tilde{f}_{\alpha m}^{n} \tilde{T}_{n}, \quad\left[H_{i}, \tilde{T}_{m}\right]=\tilde{g}_{i m}^{n} \tilde{T}_{n} .
$$

They can directly be read from (3.9). If one inserts (3.11) in the Cartan-Maurer equation (3.2) one would obtain the second-order field equations and the Bianchi identities of the original fields in terms of the original and dual fields which are the Lagrange multipliers $[2,18]$. One can use the twisted self-duality equation which $(3.11)$ obeys and which gives the first-order equations to eliminate the dual fields and then write the second-order field equations solely in terms of the original fields namely one would reach (2.12). This is analogous to what we have done in the derivation of the algebra structure. We have obtained the second-order field equations in terms of the structure constants of the algebra by inserting (3.5) in (3.2) and then we have compared the result with (2.12) to read the structure constants. The second-order field equations in terms of the structure constants that are mentioned above do not contain the dual, Lagrange multiplier fields since we have used primarily the twisted self-duality condition that relates the dual fields to the original ones and we have written the Cartan form $\mathcal{G}^{\prime}$ only in terms of the original fields in (3.5).

Since we have obtained the explicit form of the Cartan form $\mathcal{G}^{\prime}$ in (3.11) we can use the twisted self-duality equation $* \mathcal{G}^{\prime}=\mathcal{S G}^{\prime}$ to find the first-order field equations of the Lagrangian (2.10). The validity of the twisted self-duality equation is justified in the way that we have primarily assumed that $\mathcal{G}^{\prime}$ obeys it when we derived the structure constants which are chosen such that they give the correct Cartan form $\mathcal{G}^{\prime}$ which leads to the secondorder field equations (2.12) in (3.2). Therefore directly from (3.11) the twisted self-duality equation $* \mathcal{G}^{\prime}=\mathcal{S} \mathcal{G}^{\prime}$ yields

$$
\begin{aligned}
& v_{l}^{k} * d A^{l}=(-1)^{m(D-m)+1}\left(\left(v^{T}\right)^{-1}\right)_{l}^{k} d \tilde{A}^{l}, \\
& e^{\frac{1}{2} \alpha_{i} \phi^{i}}(\boldsymbol{\Omega})_{l}^{\alpha+r} * d \chi^{l}=(-1)^{D}\left(e^{\boldsymbol{\Gamma}} e^{\boldsymbol{\Lambda}}\right)_{j}^{\alpha+r} \tilde{\mathbf{S}}^{j}, \\
& \frac{1}{2} * d \phi^{i}=(-1)^{D}\left(e^{\boldsymbol{\Gamma}} e^{\boldsymbol{\Lambda}}\right)_{j}^{i} \tilde{\mathbf{S}}^{j}+(-1)^{m(D-m)+D} \frac{1}{4}\left(H_{i}\right)_{k l} A^{k} \wedge d \tilde{A}^{l} .
\end{aligned}
$$

The exterior differentiation of (3.14) gives the second-order field Eq. (2.12) indeed. We should remark once more that the roots in $\Delta_{\mathrm{nc}}^{+}$and the corresponding generators $\left\{E_{\alpha}\right\}$ are enumerated. We can also express Eq. (3.14) in a more compact form as

$$
\begin{aligned}
& \mathcal{M} * \overrightarrow{\mathbf{d A}}=(-1)^{m(D-m)+1} \overrightarrow{\mathbf{d} \tilde{\mathbf{A}}} \\
& * \vec{\Psi}=\overrightarrow{\mathbf{P}}+(-1)^{D} e^{\Gamma} e^{\boldsymbol{\Lambda}} \overrightarrow{\tilde{\mathbf{S}}}
\end{aligned}
$$


where we define the column vector $\vec{\Psi}$ as

$$
\begin{aligned}
& \boldsymbol{\Psi}^{i}=\frac{1}{2} d \phi^{i} \quad \text { for } i=1, \ldots, r, \\
& \boldsymbol{\Psi}^{\alpha+r}=e^{\frac{1}{2} \alpha_{i} \phi^{i}} \boldsymbol{\Omega}_{l}^{\alpha} d \chi^{l} \quad \text { for } \alpha \in \Delta_{\mathrm{nc}}^{+} .
\end{aligned}
$$

Also the vector $\overrightarrow{\mathbf{P}}$ is

$$
\begin{aligned}
& \mathbf{P}^{i}=(-1)^{m(D-m)+D} \frac{1}{4}\left(H_{i}\right)_{k l} A^{k} \wedge d \tilde{A}^{l} \text { for } i=1, \ldots, r, \\
& \mathbf{P}^{\alpha+r}=0 \quad \text { for } \alpha \in \Delta_{\mathrm{nc}}^{+} .
\end{aligned}
$$

\section{Conclusion}

After a concise discussion of the symmetric space sigma model with its algebraic background we have defined the coupling of $m$-form field strengths to the scalar Lagrangian in Section 2. We have also obtained the field equations following the outline of $[16,17]$. In Section 3 we have adopted the dualisation method of [1,2] to establish a coset formulation of the theory and to explore the Lie superalgebra which leads to the first-order equations of motion as a twisted self-duality condition. The validity of the twisted self-duality property of the Cartan form is implicitly justified by our construction of the algebra since beside using the second-order field equations and the Cartan-Maurer equation we have also assumed that the Cartan form obeys the twisted self-duality equation in expressing it only in terms of the original fields during the derivation of the structure constants of the algebra. As a result we have constructed a coset element by defining a Lie superalgebra structure and we have shown that both the first and the second-order field equations can be directly obtained from the Cartan form of the coset element.

This work can be considered as an extension of the results which are obtained in [15]. The dualisation of the $G / K$ symmetric space sigma model is performed in [15] when the global symmetry group is a non-split semi-simple real form. Here we have studied the dualisation of the non-split scalar coset when it is coupled to other matter fields. We have constructed a framework in which the dualisation analysis of [15] is improved to include the coupling matter fields. As a result we have obtained a general scheme which can be effectively used in the coset realizations of the whole set of matter coupled supergravities.

The formulation given in this work assumes a general non-split scalar coset $G / K$ in $D>2$ spacetime dimensions. The coupling potentials are assumed to be $(m-1)$-forms. As it is clear from the construction, the results are general and they are applicable to a wide class of supergravity theories which contain similar couplings. In [19] the bosonic sector of the ten-dimensional simple supergravity which is coupled to $N$ Abelian gauge multiplets is compactified on the Euclidean tori $T^{10-D}$ and the resulting theories in various dimensions have scalar cosets with couplings based on global symmetry groups which are non-compact real forms of some semi-simple Lie groups. Therefore the results presented here are applicable on them.

One can improve the dualized coset formulation presented here by including the gravity and the Chern-Simons terms as well. This would extend the algebra structure obtained 
here. The group theoretical aspects of the coset formulation and the symmetry properties of the first-order equations which are not considered in this work also need to be examined. One can also study the Kac-Moody symmetry scheme [3-5] of the matter coupled scalar cosets.

\section{References}

[1] E. Cremmer, B. Julia, H. Lü, C.N. Pope, Dualisation of dualities, Nucl. Phys. B 523 (1998) 73, hepth/9710119.

[2] E. Cremmer, B. Julia, H. Lü, C.N. Pope, Dualisation of dualities II: Twisted self-duality of doubled fields and superdualities, Nucl. Phys. B 535 (1998) 242, hep-th/9806106.

[3] P.C. West, Hidden superconformal symmetry in M-theory, JHEP 0008 (2000) 007, hep-th/0005270.

[4] P.C. West, E(11) and M-theory, Class. Quantum Grav. 18 (2001) 4443, hep-th/0104081.

[5] I. Schnakenburg, P.C. West, Kac-Moody symmetries of IIB supergravity, Phys. Lett. B 517 (2001) 421, hep-th/0107181.

[6] I.C.G. Campbell, P.C. West, $N=2, D=10$ nonchiral supergravity and its spontaneous compactification, Nucl. Phys. B 243 (1984) 112.

[7] M. Huq, M.A. Namazie, Kaluza-Klein supergravity in ten dimensions, Class. Quantum Grav. 2 (1985) 293.

[8] F. Giani, M. Pernici, $N=2$ supergravity in ten dimensions, Phys. Rev. D 30 (1984) 325.

[9] J.H. Schwarz, P.C. West, Symmetries and transformations of chiral $N=2, D=10$ supergravity, Phys. Lett. B 126 (1983) 301.

[10] P.S. Howe, P.C. West, The complete $N=2, D=10$ supergravity, Nucl. Phys. B 238 (1984) 181.

[11] J.H. Schwarz, Covariant field equations of chiral $N=2, D=10$ supergravity, Nucl. Phys. B 226 (1983) 269.

[12] E. Cremmer, B. Julia, J. Scherk, Supergravity theory in eleven-dimensions, Phys. Lett. B 76 (1978) 409.

[13] S. Helgason, Differential Geometry, Lie Groups and Symmetric Spaces, Graduate Studies in Mathematics, vol. 34, American Mathematical Society, Providence, RI, 2001.

[14] L. Andrianopoli, R. D’Auria, S. Ferrara, P. Fré, M. Trigiante, RR scalars, U-duality and solvable Lie algebras, Nucl. Phys. B 496 (1997) 617, hep-th/9611014.

[15] N.T. Yilmaz, The non-split scalar coset in supergravity theories, Nucl. Phys. B 675 (2003) 122, hepth/0407006.

[16] A. Keurentjes, The group theory of oxidation II: Cosets of non-split groups, Nucl. Phys. B 658 (2003) 348, hep-th/0212024.

[17] A. Keurentjes, The group theory of oxidation, Nucl. Phys. B 658 (2003) 303, hep-th/0210178.

[18] C.N. Pope, Lecture Notes on Kaluza-Klein Theory, unpublished.

[19] H. Lü, C.N. Pope, K.S. Stelle, M-theory/heterotic duality: a Kaluza-Klein perspective, Nucl. Phys. B 548 (1999) 87, hep-th/9810159. 